\title{
Spinal Sarcoidosis Presenting with Epiconus Syndrome
}

\author{
Atsushi Tsuchiya ${ }^{1}$, Hisanao Akiyama ${ }^{2}$ and Yasuhiro Hasegawa ${ }^{2}$
}

\begin{abstract}
A spinal cord lesion is a rare manifestation of neurosarcoidosis. We herein report a case with dysuria and gradually worsening numbness in both lower extremities. Thoracic magnetic resonance imaging (MRI) revealed a long spinal cord lesion with gadolinium enhancement in the lower part of the lesion. Chest computed tomography demonstrated enlarged hilar lymph nodes, and a biopsy revealed sarcoidosis. Methylprednisolone pulse therapy was started, but a follow-up MRI showed aggravation of the spinal lesion. After the addition of methotrexate to the therapy, the patient's aggravation of the spinal lesion and clinical symptoms ceased. Early diagnosis and treatment using combined methylprednisolone and methotrexate therapy may improve this devastating spinal cord disease.
\end{abstract}

Key words: spinal sarcoidosis, magnetic resonance imaging (MRI), immunosuppressive therapy

(Intern Med 53: 2529-2532, 2014)

(DOI: 10.2169/internalmedicine.53.1685)

\section{Introduction}

Sarcoidosis is a systemic non-caseating granulomatous disease of unknown etiology resulting in various dysfunctions in multiple organs. However, involvement of the nervous system in the form of neurosarcoidosis is observed in only about $5 \%$ of cases, and involvement of the spinal cord is more rare $(1,2)$. We herein describe a patient with spinal sarcoidosis exhibiting a long edematous spinal cord lesion from the Th1 to L1 level on the magnetic resonance imaging (MRI), which was successfully treated with the combination of methylprednisolone and methotrexate.

\section{Case Report}

A 69-year-old man presented to our hospital in December, 2012 with progressive numbness in both of his lower extremities and dysuria. He had been treated for diabetes and hypertension since the age of 40 years. His dysuria had also been treated since April 2012 by an urologist. Lumbar radiography and the MRI showed no abnormalities. However, the patient's symptoms gradually worsened and he reported numbness in the lower body up to his navel.

On admission, the symmetrical disturbance of vibration sense and numbness without muscle weakness were observed in both lower extremities. Hyper-reflexia and the Chaddock and Babinski signs were also observed in both lower extremities and the patient's gait was unsteady. The laboratory findings, including the blood analysis and serum biochemistry findings, were normal. The serum concentrations of the angiotensin-converting enzyme and ionized calcium were also normal. A variety of antibodies, such as anti-nuclear, anti-double-stranded DNA, anti-Smith, anticardiolipin, anti-SS-A, anti-SS-B, anti-ribonucleoprotein (RNP), and anti-aquaporin 4, were all negative. The cerebrospinal fluid examination revealed 10 leukocytes $/ \mathrm{mm}^{3}$ accompanied by high protein $(155 \mathrm{mg} / \mathrm{dL})$ and normal glucose levels $(75 \mathrm{mg} / \mathrm{dL})$. Unfortunately, the CD4/CD8 ratio was not measured and bronchoalveolar lavage and fluorodeoxyglucose positron emission tomography (FDG-PET) were not performed. The tuberculin reaction was positive at a diameter of $12 \mathrm{~mm}$.

The T2-weighted MRI performed on admission revealed a long, edematous spinal cord lesion extending from the Th1 to L1 level with signal hyperintensity. Gadolinium enhancement was observed from the Th9 to Th10 level (Fig. 1). Chest computed tomography demonstrated enlargement of the bilateral hilar lymph nodes and diffuse nodules in both lung fields (Fig. 2). Ga-67 scintigraphy findings were nega-

${ }^{1}$ Department of Neurology, St. Marianna University School of Medicine Yokohama City Seibu Hospital, Japan and ${ }^{2}$ Department of Neurology, St. Marianna University School of Medicine, Japan

Received for publication September 1, 2013; Accepted for publication April 14, 2014

Correspondence to Dr. Atsushi Tsuchiya, a-t1@ marianna-u.ac.jp 

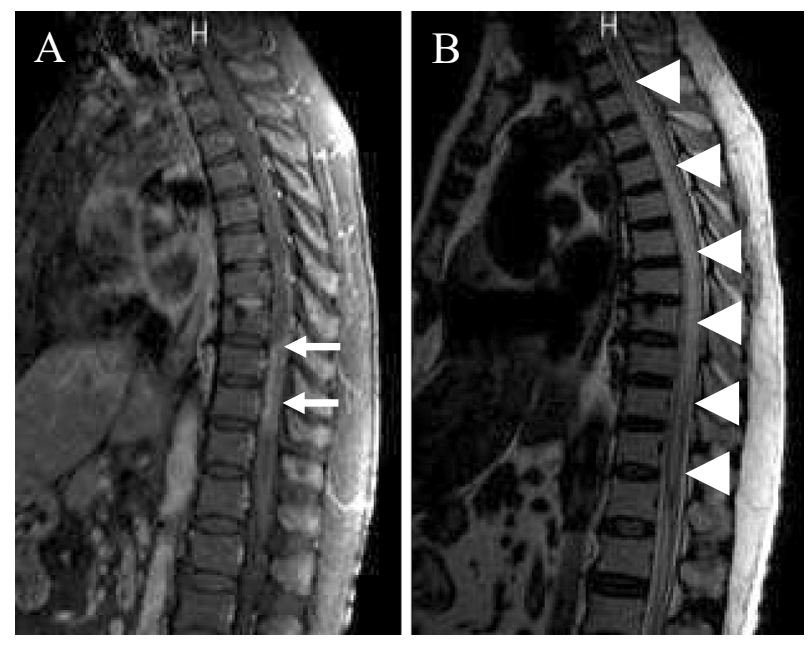

Figure 1. Thoracic magnetic resonance image (MRI). (A) Gadolinium-enhanced T1-weighted sagittal imaging on admission (1.5 T; TR, $550 \mathrm{~ms}$; TE, $11.1 \mathrm{~ms})$ shows enhancement at the Th9-Th10 level (arrows). (B) The T2-weighted sagittal imaging on admission (1.5 T; TR, 4,000 ms; TE, $106 \mathrm{~ms}$ ) shows a linear, consecutive edematous signal hyperintensity at the Th1L1 level (arrowheads).

tive for the lesions. Nerve conduction studies and electromyography also showed no abnormalities. The short-latency somatosensory evoked potentials (SEP) demonstrated the disappearance of peaks after the P24 component, thus suggesting an intramedullary lesion in the epiconus.

An ophthalmological examination revealed no abnormalities in the conjunctiva, uvea, or fundus. Echocardiography yielded normal results. For further examination, we also performed an endoscopic biopsy of a hilar lymph node.

Dysuria, hypoesthesia and paresthesia in the lower extremities gradually worsened. Three weeks after admission, the patient's thermal nociception dropped to $2 / 10$, the vibration sensation disappeared in both lower extremities, and self-catheterization was required for dysuria. A walker was also necessary for mobility. Although the pathological diagnosis remained unclear, intravenous methylprednisolone pulse therapy followed by oral prednisolone was started due to the aggravation of the patient's symptoms. After the steroid treatment, the MRI findings improved slightly (Fig. 3) and his clinical symptoms ceased. However, the disappearance of peaks in SEP was unchanged.

A histological examination of the hilar lymph node revealed a non-caseating epithelioid granuloma (Fig. 4) and sarcoidosis was strongly suspected. A follow-up MRI performed 8 weeks after admission demonstrated further worsening of the spinal cord lesion without symptom aggravation. Methotrexate therapy was therefore increased to 10 $\mathrm{mg} /$ week as a maintenance dose. After initiating this treatment, the aggravation of the MRI findings and his clinical symptoms ceased (Fig. 5). Prednisolone (20 mg/day) and methotrexate $(10 \mathrm{mg} /$ week) treatment was continued for 12 weeks, and the progression of the patient's clinical symptoms stopped.
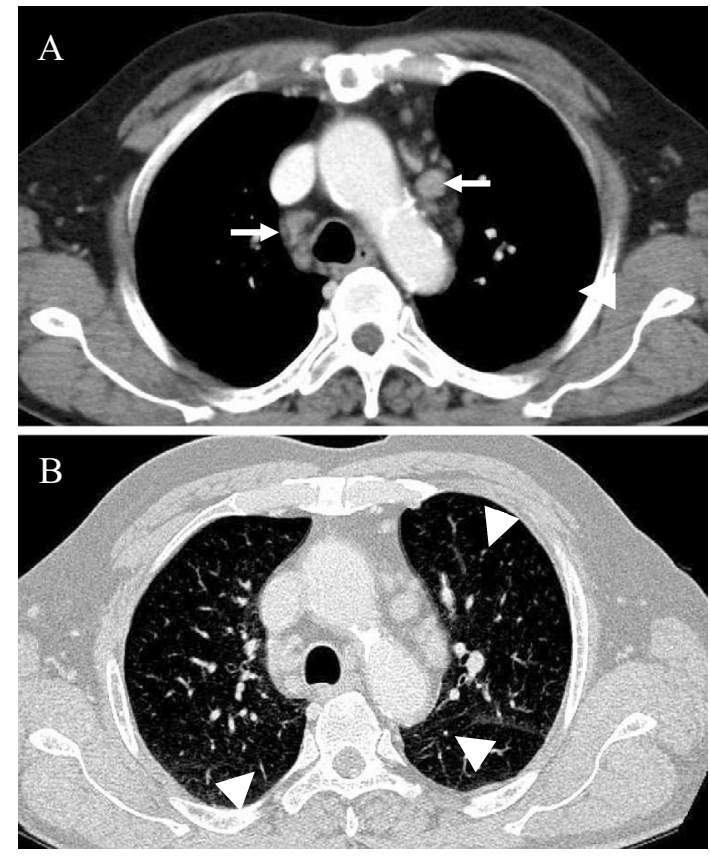

Figure 2. Chest computed tomography (CT). Chest CT performed on admission shows enlargement of (A) the bilateral hilar lymph nodes (arrows) and (B) diffuse micronodules in the bilateral lungs (arrowheads).

\section{Discussion}

Neurosarcoidosis is a term for sarcoidosis involving the nervous system. The incidence of this pathology is very low, seen in $5-6 \%$ of cases of sarcoidosis $(3,4)$. A predominance of female patients was observed in a case series of 166 patients with neurosarcoidosis in Japan. Iizuka et al. (5) classified neurosarcoidosis into five types based on the imaging and clinical features: 1) intramedullary granuloma lesion; 2) meningeal lesion; 3) hydrocephalus; 4) vascular lesion; and (2) encephalopathy. Neurosarcoidosis may present with various neurological symptoms. Because the cranial nerves, the hypothalamus, and the hypophysis are usually involved in neurosarcoidosis, symptoms related to the cranial nerves or a hypophyseal lesion are common (4). Spinal cord lesions are extremely rare, seen in only $6-8 \%$ of cases of neurosarcoidosis (2), but this possibility should not be overlooked because it may lead to poor functional outcomes. An isolated spinal cord lesion was observed in only $16 \%$ of spinal cord sarcoidosis (1). Several case reports have so far been published, but no case series has yet been reported from Japan. The incidence by location of the sarcoid granuloma has been reported as: intramedullary, 35\%; extra-medullary intradural, 35\%; a combination of these two compartments, 23\%; and extradural, 7\% (6). The MRI findings characteristically depict a long, edematous spinal cord lesion with signal hyperintensity on the T2-weighted imaging. The leptomeningeal enhancement can be observed in $40-67 \%$ of patients with spinal cord sarcoidosis (7). Although the spinal cord lesion extended from the cervical to lumbar segment, the neurological deficits were limited in both lower extremi- 

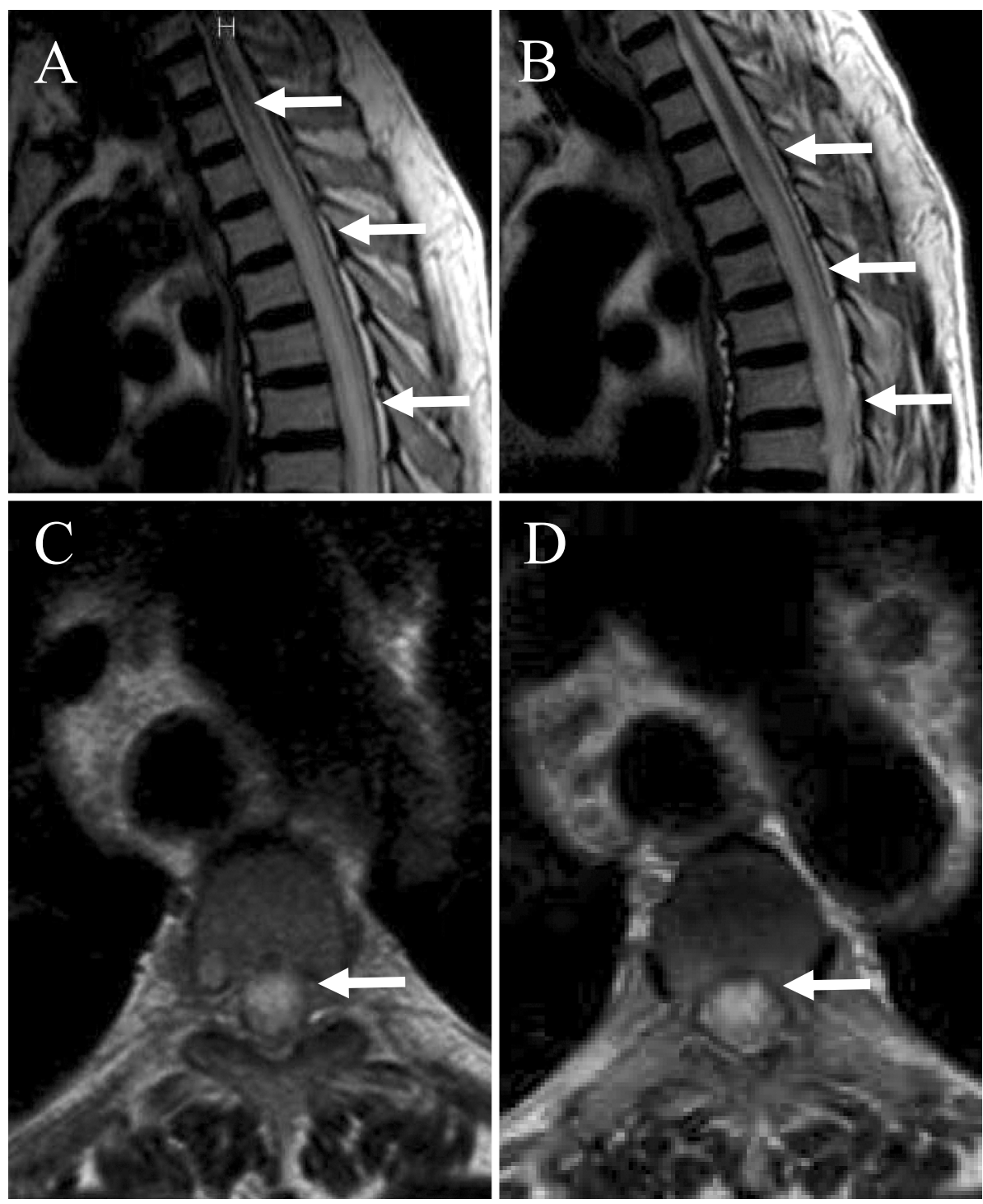

Figure 3. The T2-weighted sagittal and axial imagings on admission (A, C) and after 6 weeks (B, D). A consecutive, long segmental and edematous hyperintensity was shortened between the sagittal images ( $\mathrm{A}$ and $\mathrm{B})$ and was reduced between the axial images $(\mathrm{C}$ and $\mathrm{D})$.

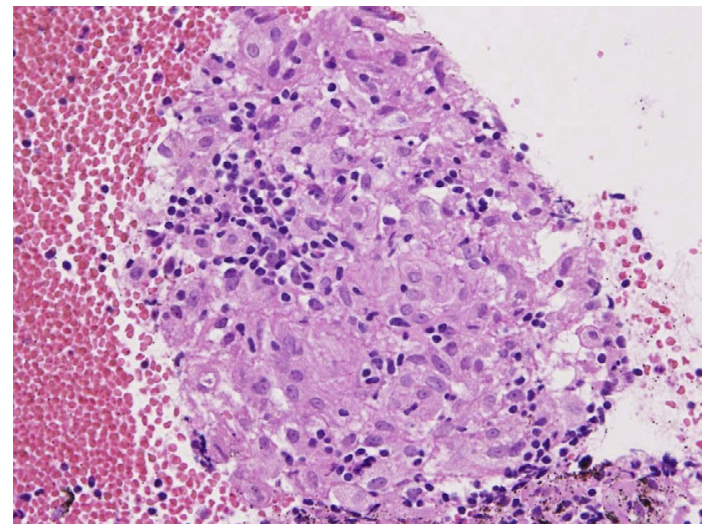

Figure 4. The pathological findings of the biopsied hilar lymph node. The pathological findings revealed a non-caseating epithelioid granuloma (Hematoxylin and Eosin staining).

ties, which could have resulted from the enhanced lesion on the MRI. According to the MRI staging by Junger et al. (2), the present case could be classified as stage 2 , for which a good prognosis can be expected if treatment is initiated early. Corticosteroids are considered the first-line agent, and oral prednisolone is usually started at a dose of 40-60 mg/ day, then gradually tapered $(1,8,9)$. However, Lower et al. reported that only $29 \%$ of patients with neurosarcoidosis respond to steroids alone (10), and non-responders to steroid therapy or cases that show difficulty in decreasing the steroid dose are advised to receive combination therapy with an immunosuppressant at an early stage (11). In this case, the steroid pulse therapy was followed by oral steroid therapy. Although a slight improvement in the MRI lesion size was seen, the patient's symptoms deteriorated with the gradual decrease in oral steroid use. When we administered additional immunosuppressive therapy with methotrexate, the disease progression stopped.

In an extensive review of the literature, $66 \%$ of patients with spinal sarcoidosis improved, 25\% deteriorated, and $9 \%$ remained stable (1). Spontaneous remission of the clinical symptoms is not seen in spinal sarcoidosis, and patients 


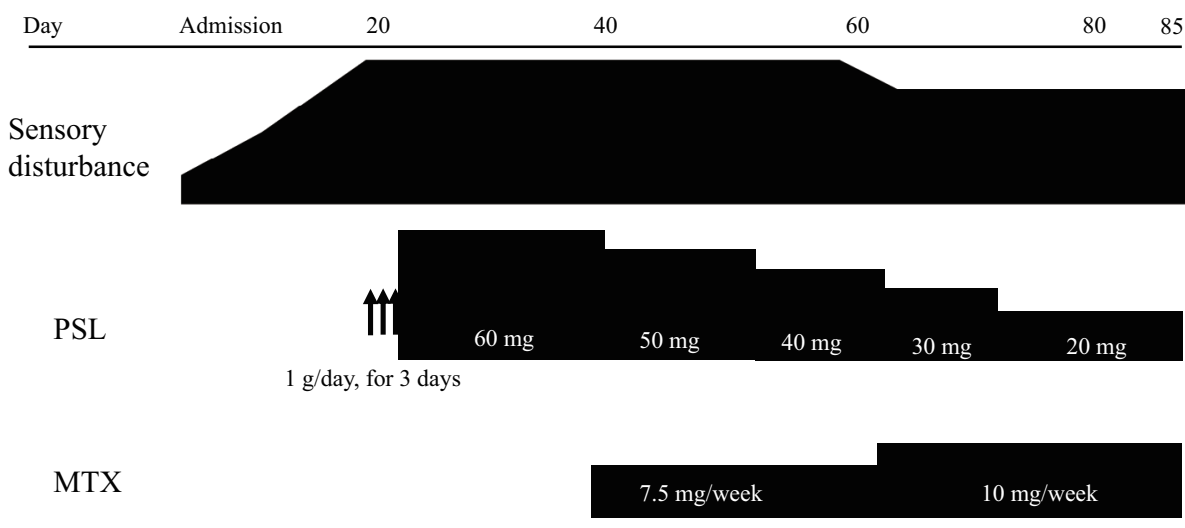

Figure 5. The clinical course. PSL: prednisolone, MTX: methotrexate

treated before the onset of severe neurological deficits tend to experience better outcomes (8). No guidelines or consensus have yet been reached regarding when additional immunosuppressive therapy should be started. Varron at el. recommend steroid as the first-choice treatment, and methotrexate can be added for patients without any response to steroid therapy or with severe symptoms. Azathioprine or infliximab in conjunction with an immunosuppressant could be used as second-line agents for patients with ineffective therapy (11). Early diagnosis of neurosarcoidosis is important for a good prognosis. However, it is important to note that the present case is not considered typical forneurosarcoidosis (12). In order to diagnose neurosarcoidosis by the diagnostic criteria, a clinical presentation suggestive of neurosarcoidosis must be seen, along with the pathological confirmation of systemic sarcoidosis and positive test results for at least two of the fixed 6 laboratory findings. Although only 1 of the laboratory findings was fulfilled in our case, we initiated treatment for a diagnosis of neurosarcoidosis because we had already excluded all other diseases as the cause of the spinal lesion. If we adhere strictly to the diagnostic criteria, the early treatment of neurosarcoidosis may be delayed; therefore, flexible judgment is required in cases such as ours which cannot be easily examined by an openbiopsy of the spinal cord. The suspicion of this rare disease as a differential diagnosis and thorough work-up of other organs for which one can obtain biopsy specimens is warranted.

We herein described a case of spinal sarcoidosis presenting with epiconus syndrome. Spinal lesions are a rare manifestation of sarcoidosis and they are difficult to diagnosis due to non-specific findings. Early diagnosis and treatment initiation can improve the prognosis. However, no standard treatment regimen for spinal sarcoidosis has yet been established and the effects of each medication are not fixed. Therefore, the combination of methylprednisolone and methotrexate represents one choice of treatment to improve this devastating spinal cord disease.

The authors state that they have no Conflict of Interest (COI).

\section{References}

1. Saleh S, Saw C, Marzouk K, Sharma O. Sarcoidosis of the spinal cord: literature review and report of eight cases. J Natl Med Assoc 98: 965-976, 2006

2. Junger SS, Stern BJ, Levine SR, Sipos E, Marti-Masso JF. Intramedullary spinal sarcoidosis: clinical and magnetic resonance imaging characteristics. Neurology 43: 333-337, 1993.

3. Chapelon C, Ziza JM, Piette JC, et al. Neurosarcoidosis: signs, course and treatment in 35 confirmed cases. Medicine (Baltimore) 69: 261-276, 1990.

4. Stern BJ, Krumholz A, Johns C, Scott P, Nissim J. Sarcoidosis and its neurological manifestations. Arch Neurol 42: 909-917, 1985.

5. Iizuka T, Iigaya M, Tsukahara S. Nervous system diseases associated with sarcoidosis. Nihon Naika Gakkai Zasshi (The Journal of the Japanese Society of Internal Medicine) 88: 808-817, 1999 (in Japanese).

6. Hayat GR, Walton TP, Smith KR Jr, Martin DS, Manepalli AN. Solitary intramedullary neurosarcoidosis: role of MRI in early detection. J Neuroimaging 11: 66-70, 2001.

7. Lexa FJ, Grossman RI. MR of sarcoidosis in the head and spine: spectrum of manifestations and radiographic response to steroid therapy. AJNR Am J Neuroradiol 15: 973-982, 1994.

8. Bradley DA, Lower EE, Baughman RP. Diagnosis and management of spinal cord sarcoidosis. Sarcoidosis Vasc Diffuse Lung Dis 23: 58-65, 2006.

9. Terushkin V, Stern BJ, Judson MA, et al. Neurosarcoidosis: presentations and management. Neurologist 16: 2-15, 2010.

10. Lower EE, Broderick JP, Brott TG, Baughman RP. Diagnosis and management of neurological sarcoidosis. Arch Intern Med 157: 1864-1868, 1997.

11. Varron L, Broussolle C, Candessanche JP, et al. Spinal cord sarcoidosis: report of seven cases. Eur J Neurol 16: 289-296, 2009.

12. Tsuda T. Diagnostic standard and guideline for sarcoidosis- 2006 . Sarcoidosis/Nikugashuseishikkan (The Japanese Journal of Sarcoidosis and Other Granulomatous Disorders) 26: 77-82, 2006 (in Japanease).

(C) 2014 The Japanese Society of Internal Medicine http://www.naika.or.jp/imonline/index.html 